\title{
Cardiac toxicity of immune-checkpoint inhibitors: a clinical case of nivolumab-induced myocarditis and review of the evidence and new challenges
}

This article was published in the following Dove Press journal: Cancer Management and Research

\author{
Roberto Martin Huertas' \\ Cristina Saavedra Serrano' \\ Cristian Perna ${ }^{2}$ \\ Ana Ferrer Gómez ${ }^{2}$ \\ Teresa Alonso Gordoa' \\ 'Medical Oncology Department, \\ Universitary Hospital Ramon y Cajal, \\ Madrid, Spain; ${ }^{2}$ Pathology Department, \\ Universitary Hospital Ramon y Cajal, \\ Madrid, Spain
}

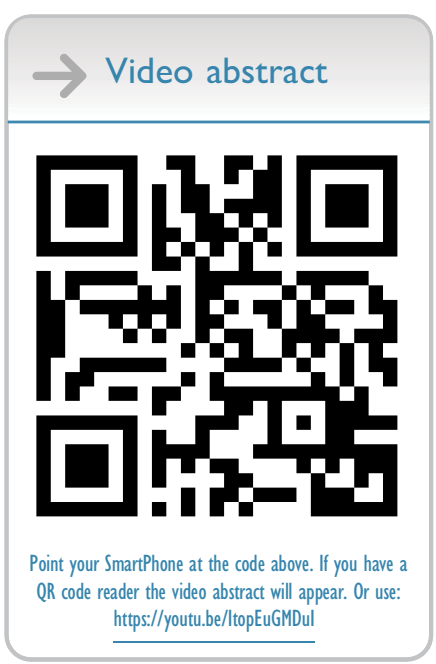

Correspondence: Teresa Alonso Gordoa Medical Oncology Department, Universitary Hospital Ramon y Cajal, Ctra., Colmenar Km 9100, Madrid 28034, Spain

Email talonso@oncologiahrc.com

\begin{abstract}
Immune checkpoint inhibitors have revolutionized cancer treatment due to their undeniable efficacy, but a range of new adverse events (AE) has emerged. In particular, cardiac toxicity is a potentially fatal $\mathrm{AE}$, and introduces new challenges regarding its underlying molecular mechanisms of occurrence, optimal treatment and follow up, and prevention. We present a clinical case of a patient with advanced kidney cancer treated with nivolumab as a third line treatment. After four cycles, the patient developed nonspecific symptoms and was hospitalized, identifying a set of clinical, analytical and electrocardiographic alterations compatible with myocarditis. Despite the intensive support, the patient died and a necropsy study was performed. We present a detailed description of the clinical case including the pathological and molecular findings, and we conduct a review of the available evidence related to immune-mediated cardiac toxicity to offer some new highlights in the management of this AE.
\end{abstract}

Keywords: myocarditis, nivolumab, kidney, immune-related adverse events

\section{Background}

The introduction of immune checkpoint inhibitors (ICI), such as anti-programmed cell death-1 (PD1) monoclonal antibodies, has changed cancer treatment in many tumor types. However, despite their benefits, a range of new adverse events (AE) has emerged, different from those described with chemotherapy and other targeted drugs, due to the ability of these agents to elicit an autoimmune response.

Nivolumab, a fully human immunoglobulin (Ig) G4 monoclonal antibody directed against cell surface receptor PD1, has been approved for the treatment of patients with advanced renal cell carcinoma previously treated with vascular endothelial growth factor receptor (VEGFR) inhibitors, based on the survival results from the Phase III clinical trial Checkmate 025. A number of AE had been reported with nivolumab treatment in this population, including hypothyroidism, pneumonitis, hepatitis, colitis, neuropathy, uveitis and dermatitis; $19 \%$ of grades 3-4 AE were described in the nivolumab group versus $37 \%$ in the control arm with no deaths recorded with nivolumab. ${ }^{1}$

Cardiac toxicity has been a relevant issue with classical antineoplastic drugs, but underestimated in initial ICI trials, particularly myocarditis. ${ }^{2}$ PD-1 and PD-L1 are expressed in human cardiomyocytes, and PD-1 deletion causes autoimmune myocarditis in xenografts. ${ }^{3-6}$ 
Over the last years, several cases of myocarditis have been reported in patients treated with ICI monotherapy or in combination (Table 1). ${ }^{7-21}$ The concern about this potentially fatal $\mathrm{AE}$ is increasing and different questions are trying to be solved concerning its incidence, underlying molecular mechanisms, optimal treatment, follow up and preventive measures.

We report the patient's case with advanced kidney cancer who developed nivolumab-related myocarditis, with a detailed description of the clinical case including pathological and molecular findings from the patient's necropsy. Finally, an exhaustive review of the available evidence related to immune-mediated cardiac toxicity to offer some new focal points in the management of this $\mathrm{AE}$ was conducted.

\section{Case report}

An 80-year-old man with no cardiovascular disease beside arterial hypertension, and no history of autoimmune disorders was treated with nivolumab as third-line treatment for advanced clear cell kidney cancer with lung metastases and abdominal subcutaneous implants.

Patient was diagnosed with renal cancer with lung metastases in 2015, starting first-line treatment with sunitinib. In 2017, after 2 years of treatment, the disease progressed with new lesions as abdominal subcutaneous implants, so secondline with axitinib was introduced. However, 3 months later, an increase in the abdominal implants size was identified and we began a third-line treatment with nivolumab. Therefore, the patient was a long time responding to first-line antiangiogenic agent (sunitinib), but early progressor to a second tyrosine kinase inhibitor (TKI).

After four cycles of nivolumab (more than 2 months of the initial dose), the patient was admitted to our hospital due to a severe asthenia and poor pain control related to subcutaneous tumor infiltration, with no typical symptoms of angina pectoris. Initial work-up revealed previously unknown atrial fibrillation and left bundle branch block in the electrocardiogram (ECG; Figure 1). As well as altered cardiac damage parameters, such as elevated levels of creatine kinase (CK) of 1,853 U/L (normal range (NR) 38-174 U/L), troponin I (TnI) of $19.4 \mathrm{ng} / \mathrm{mL}(\mathrm{NR}<0.1 \mathrm{ng} /$ $\mathrm{mL}$ ) and brain natriuretic peptide (BNP) of $1,413 \mathrm{pg} / \mathrm{mL}$ (NR $<100 \mathrm{pg} / \mathrm{mL}$ ). Moreover, reactive $\mathrm{C}$ protein $(\mathrm{RCP})$ was elevated $(151,8 \mathrm{mg} / \mathrm{L},[\mathrm{NR}<5 \mathrm{mg} / \mathrm{L}])$ and lymphopenia of 670 lymphocytes was seen $(\mathrm{NR}>1,000 / \mu \mathrm{L})$.

Due to these alterations, an urgent transthoracic echocardiogram (TTE) was performed, with no change from the one performed 4 months earlier (preserved left ventricular systolic function with mild concentric hypertrophy), although with dyssynchrony.

We suspected nivolumab-induced myocarditis, so highdose glucocorticoids (GC) were initiated $(2 \mathrm{mg} / \mathrm{kg} /$ day intravenous methylprednisolone). In the subsequent analytical control, CK, TnI and PCR levels dropped $(1,275 \mathrm{U} / \mathrm{L}$, $14 \mathrm{ng} / \mathrm{mL}$ and $108.3 \mathrm{mg} / \mathrm{L}$, respectively).

Additional work-up was performed. He had no symptoms suggestive of viral illness, and serologies were negative for hepatitis B, hepatitis C, HIV, varicella-zoster virus, EpsteinBarr virus, cytomegalovirus and parvovirus. Moreover, the serologies of bacteria that could potentially cause myocarditis or cardiovascular diseases (brucella, treponema pallidum, leptospira, borrelia, rickettsia) were negative. We requested cardiological evaluation, and they reported that the clinical presentation was not suggestive of ischemia. A new TTE was performed 4 days after admission and left ventricular systolic function was slightly diminished (50\%).

The patient had no history of autoimmune disorders before nivolumab treatment but, in the diagnostic evaluation focusing on asthenia and muscular weakness, elevation of antibodies against the acetylcholine receptor was identified $(2.06 \mathrm{nmol} / \mathrm{mL}, \mathrm{NR}<0.45 \mathrm{nmol} / \mathrm{mL})$ compatible with immunological diagnosis of myasthenia gravis (MG), which happened concurrently with myocarditis. Antibodies against striated muscle were also positive. Other antibodies (antinuclear antibodies, anti-smooth muscle antibodies) were negative.

Therefore, viral infections and ischemia were ruled out as causes of myocarditis, but with the increase of antiacetylcholine antibodies.

Despite the intensive support therapy in Oncology and Cardiology units, the patient suffered a heart attack a week after being admitted in our hospital and passed away. He was diagnosed with an atrial fibrillation at myocarditis diagnosis, but at the time of death, there were no previous malignant arrhythmias or data of cardiac failure identified. In order to establish a definitive diagnosis, a necropsy was requested to the Pathology Department. A macroscopic examination disclosed metastatis found in the lung, previously diagnosed by imaging test, but it also showed multiple metastases in the liver, left kidney and peritoneum. In addition, a mass located in right retroperitoneum measuring $24 \mathrm{~cm}$ was observed. Microscopic examination of all nodules disclosed epithelial proliferation with nests, trabeculae and glands formed by characteristic clear cells of renal cell carcinoma. Nuclei were graded as Furhman 


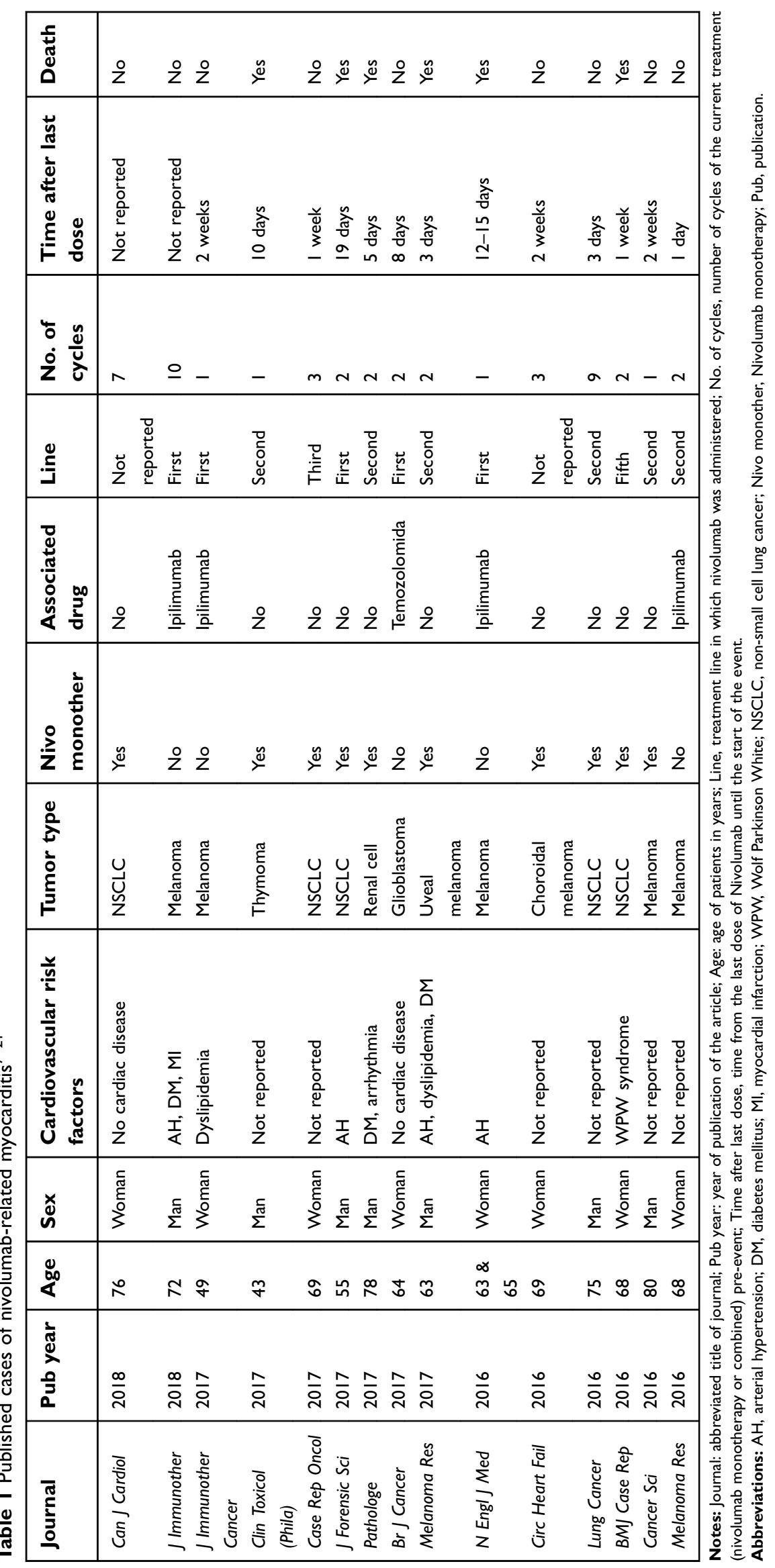



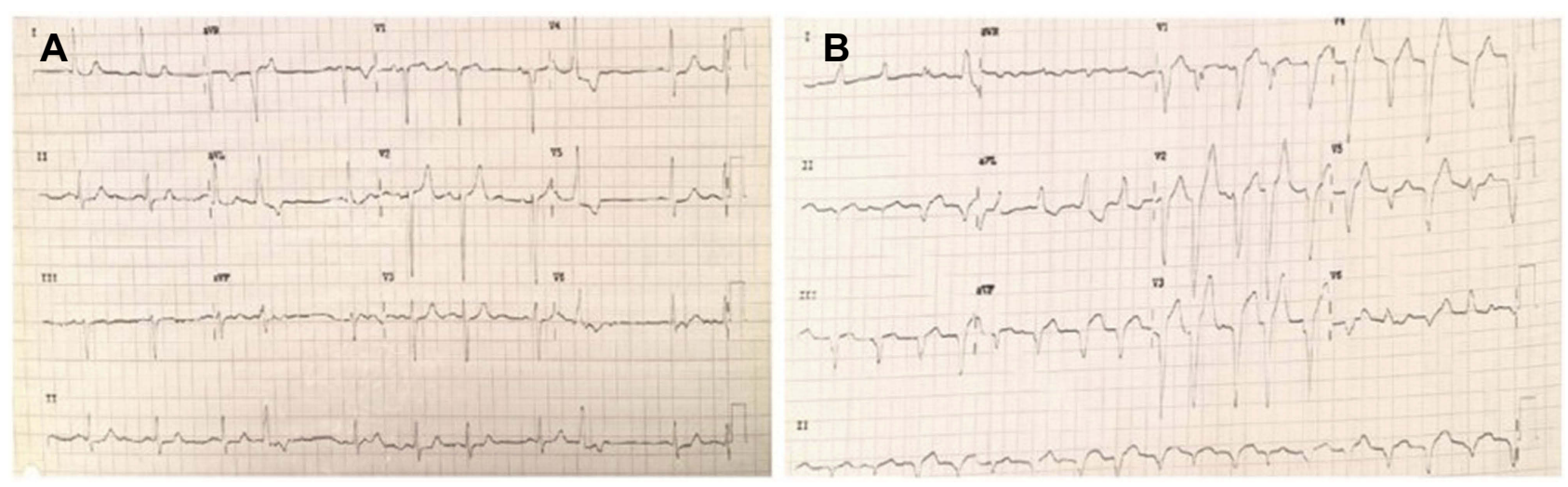

Figure I ECG baseline before starting nivolumab treatment: sinus rhythm at 60 bpm with isolated extrasystoles (A). ECG at myocarditis clinical onset: atrial fibrillation and left bundle branch block (B).

III-IV. No lymphocytic infiltrates were observed inside or in surrounding neoplastic areas, excepting lung metastases with scarce lymphoid cells, grossly the heart was normal. However, histopathological examination revealed multiple foci of myocardial lesion that involved $25-30 \%$ of the myocardium. Foci showed an intense inflammatory infiltrate and myocardial necrosis. The infiltrate was mostly composed of lymphocytes, which were entirely positive for T-cell marker CD3. The ratio of CD8/CD4 cells was $80 \% / 20 \%$, and $40 \%$ of all lymphocytes were PD-1 positive (Figure 2). CD20 was negative. There were some macrophage $\mathrm{CD} 68+$ cells associated to necrotic myocardial muscle. Neither neutrophilic nor eosinophilic infiltrated were observed. Myocardial fresh tissue was sent to Microbiology Department to investigate the possibility of viral infection. With this issue, all molecular tests were negative.

\section{Discussion}

ICI have considerably improved clinical outcomes in multiple tumor types and are being used both earlier and metastatic disease settings in combination with other therapies.

Treatment of metastatic clear cell renal cancer has been classically based on VEGFR TKI therapy and mammalian

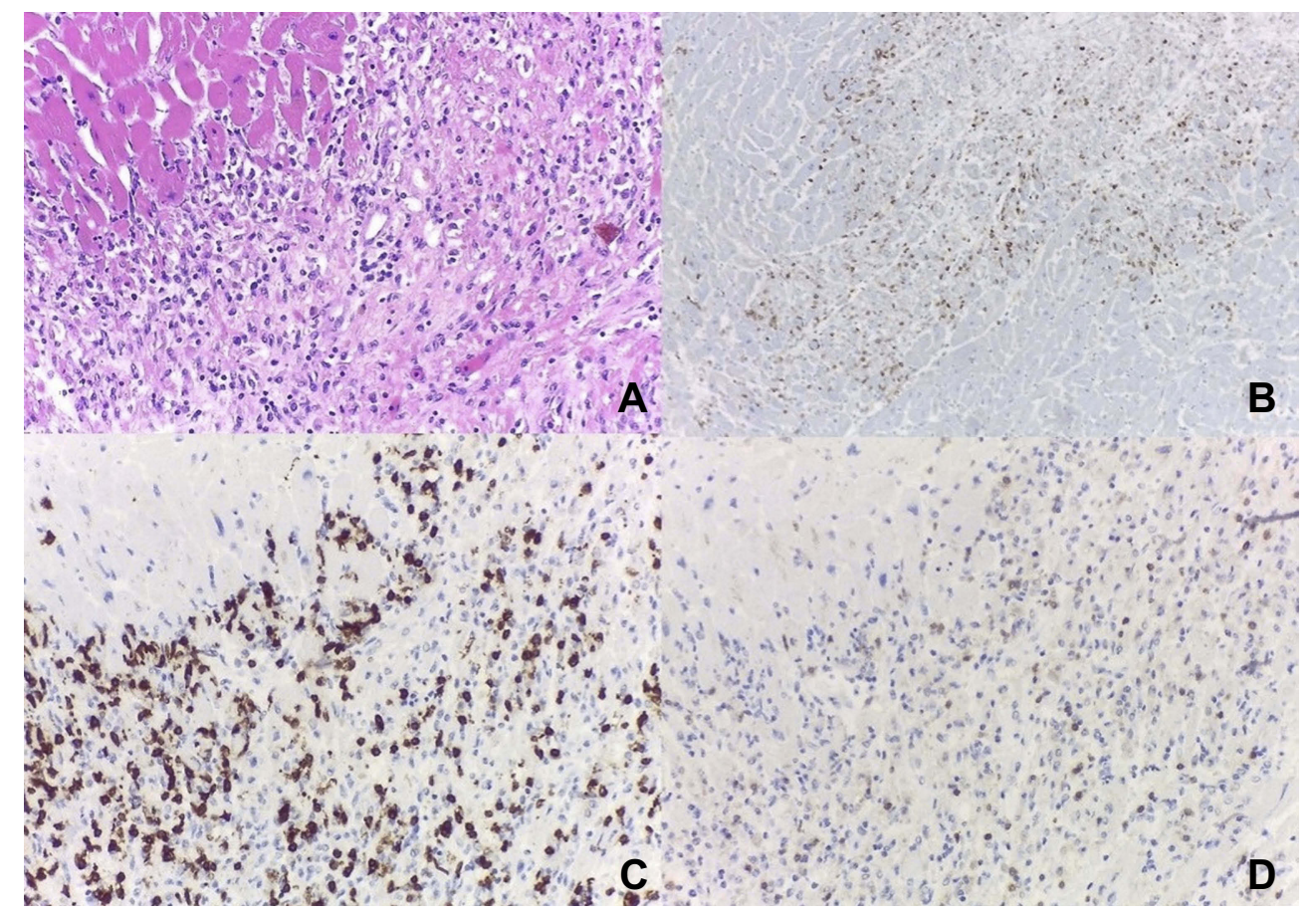

Figure 2 H\&E section of myocardial tissue with necrotic focus containing dense lymphocytic infiltrates (A); immunohistochemistry of lymphoid component disclosing PD-I cells (B), CD8 (C), and CD4 lymphocytes (D). 100x magnification. 
target of rapamycin (mTOR) inhibitors. ${ }^{22}$ Nowadays, ICI has become a standard treatment for this type of cancer. Nivolumab has been the first ICI approved for advanced renal-cell carcinoma in the second-line setting based on the results of the Phase III trial Checkmate 025, comparing nivolumab against standard treatment everolimus. The primary endpoint was achieved, with longer overall survival (25 months vs 19.6 months) and other secondary endpoints, such as overall response rate $(25 \%$ vs $5 \%){ }^{1}$

In this trial, any grade of nivolumab-related AE was observed in $79 \%$ of patients ( $88 \%$ in control arm) and grades 3-4 AE in only $19 \%$ of them (37\% in control arm). The most common AE treatment related in nivolumab arm were fatigue (33\%), nausea (14\%) and pruritus (14\%); 51\% of patients had dose delays and dose reductions were not permitted with nivolumab. Only $8 \%$ of patients discontinued treatment due to nivolumab-related $\mathrm{AE}$, no deaths were reported from drug toxic effects in nivolumab group. No cardiac toxicity was present with nivolumab.

Cardiac toxicity has been underestimated in initial ICI trials particularly myocarditis due to multiple reasons, including low incidence, early onset and non-specific symptomatology.

Myocarditis is a rare $\mathrm{AE}$, which is more frequent and severe in patients who receive combination nivolumab and ipilimumab compared with those who receive nivolumab alone $(0.27 \%$ vs $0.06 \% ; P<0.001) .{ }^{19}$ In cases of severe myocarditis, death occurs in $46 \%$ of cases, with fatality rates higher with combination than with monotherapy $(67 \%$ vs $36 \% ; P=0.008) .^{23}$

Patients have a broad spectrum of age with a median of 69 years (range $20-90$ ), and $75 \%$ of them do not report cardiovascular risk factors (CRF). Hypertension is the most frequently observed and history of diabetic medications or statin use is not usually seen (Table 1).

The characteristics of the patient that we report conform to those collected in Checkmate 025 trial. Median age was 62 years (range 18-88); 83\% had more than two metastatic sites (67\% of them were lung metastases) and $28 \%$ of patients had received two previous antiangiogenic treatments.

Moreover, age range and absence of CRF were observed in our patient as it is registered in the literature. Typically, our patient presented hypertension as the only CRF together with advanced age, which has been seen in some cases.

The most common tumor type with immune-related myocarditis are melanoma and lung cancer, although cases have also been described in renal cancer, as in our case (Table 1). ${ }^{24}$
In relation to the dose, more than a half of patients (64\%) had received one or two doses of ICI (monotherapy or combination treatment) prior to the onset of myocarditis. However, cases with more doses have also been elucidated (even after 10 cycles). Particularly with nivolumab, myocarditis has been more frequently reported after 1-3 cycles, but after more doses (7-9 cycles) this AE has also been observed. Timing of myocarditis onset from initiation of ICI treatment is typically about 2 weeks from the last received dose although in $76 \%$ of cases it occurs within the first 6 weeks of treatment (Table 1). ${ }^{23}$

Our patient received four cycles of nivolumab, and symptoms of myocarditis started 2 weeks after the last dose administered. On that same day, the patient would have received the fifth cycle, so it is similar to what was observed in other reported cases.

Non-specific symptomatology is the one of the most common manifestations of myocarditis, including fatigue, myalgias, general discomfort or nausea, like our patient who was referred to the emergency room due to severe asthenia. This is one example why the initial suspicion of myocarditis is so low since the most frequent nivolumab $\mathrm{AE}$ described in clinical trials is asthenia. In other cases, myocarditis may present with acute heart failure symptoms (dyspnea, edema of the legs) or arrhythmia. In addition to fatigue, the patient we presented had atrial fibrillation not previously known in the ECG. Interestingly, there are some cases of silent latent myocarditis detailed. ${ }^{9}$

The non-specific symptomatology together with the early appearance after the first cycles of treatment as well as the rapid progression, delay and interfere myocarditis diagnosis and its treatment. Cardiac monitoring strategy using biochemical markers (TnI, BNP) or ECG has not been validated. Since most patients develop the symptoms during the first cycles (1-3), during the first 4-6 weeks of treatment, as it is our case, it has been suggested a closer analytical controls of TnI prior to each new cycle and in the week after, due to in the majority of cases TnI elevation was seen. Since the incidence of myocarditis decreases in the later period, according to the literature, the interruption of these controls could be considered. However, it has not been shown that cardiac monitoring by means of TnI or ECG has a predictive value in the early diagnosis of myocarditis and other cardiac AE. ${ }^{24}$

In laboratory tests, TnI and $\mathrm{CK}$ elevations as well as abnormalities in ECG are observed. Cardiac function is usually preserved, as in our patient with left systolic 
function in low limit of normality $(50 \%)$, except in those cases that present heart failure symptoms, which may have an ejection fraction below normal.

Concurrent immune-related AE are observed in about 50\% of patients with myocarditis and the most common are myositis and MG. Bulbar symptoms and myasthenic crisis are observed more frequently in nivolumab-induced MG and most patients have positive anti-acetylcholine receptor antibodies, as detected in our patient. ${ }^{11,25}$ Although death cause of our patient was myocarditis, we cannot rule out the involvement of $\mathrm{MG}$ in this setting. The pathogenic role of casual finding of antiacetylcholine receptor antibodies in a patient with nivolumabinduced myocarditis is not well defined. The diagnosis of $\mathrm{MG}$ is based on bedside test (ice pack test and edrophonium test), compatible symptoms and electrophysiological studies that supplement serological test. Due to the rapid evolution of our patient, these studies could not be performed, so that compatible clinical and positive antibodies were the only sustenance to diagnose MG. Therefore, we cannot state categorically our patient presented concurrent MG with myocarditis and it is necessary to investigate what pathogenic role positive antiacetylcholine receptor antibodies play. In this fashion, false positives of these antibodies are very rare although it is possible as in polymyositis.

High-dose GC lead to a rapid decrease in markers of heart damage and improvement of symptoms. The patient experienced a decrease in TnI, CK and PCR levels after starting corticoid treatment.

There are no specific guidelines for the treatment of immune-related myocarditis and the recommended approach consists of immunosuppression and classical heart failure management. GC are the drugs more frequently used as immunosuppressive treatment, and they are recommended in the management of another immune-related AE. However, when patients have not achieved a successful response to GC, other medicaments are used. Anti-thymocyte globulin (ATG) has been classically used in the prophylaxis of graft versus host disease due to its ability to deplete $\mathrm{T}$ lymphocytes, among others. It has been reported as an effective drug against immune-related myocarditis, like giant cell myocarditis. ${ }^{26,27}$ For this reason, it has also been successfully used in immunerelated myocarditis secondary to medication. ${ }^{14,28}$ Indeed, infliximab, an anti-TNF drug, has also been considered for the treatment of myocarditis related to autoimmune or infectious diseases. ${ }^{29,30}$ Therefore, it has been used in some cases of myocarditis induced by ICI. ${ }^{16}$ With patients where myocarditis is concurrent with myasthenia crisis, nonspecific human Igs have been utilized. ${ }^{20,25}$
Unfortunately, our patient passed away. Mortality rates are high and nearly half of patients did not make it despite supportive treatments (Table 1$)^{23}$

As a rule, histopathology remains the gold standard for diagnosing myocarditis. However, compatible clinical scenario, laboratory testing, ECG, TTE and cardiovascular magnetic resonance features may diagnose myocarditis in most patients, biopsy is only performed in selected patients. In our case, myocarditis diagnose was based on non-specific symptoms, altered cardiac damage parameters and arrhythmia previously unknown in ECG. Due to the poor general condition of the patient, cardiovascular magnetic resonance and cardiac catheterization were not performed. Subsequently, necropsy was demanded to confirm this high suspicion.

Microscopic examination revealed an important percentage of myocardial damage, so correlation with a noninvasive test was established. Moreover, histopathological study showed that myocardial inflammation was entirely composed of $\mathrm{T}$ lymphocytes. In this context, both viral myocarditis and nivolumab-induced myocarditis might be the cause of myocardial damage. Molecular testing performed in fresh myocardial tissue showed no viral DNA.

Previous reported cases of nivolumab-induced myocarditis have shown marked CD8 cells preponderance similar to the present case. To understand this pathological finding, it is necessary to know that PD1 blockade increases the infiltrating tumor CD8+ T lymphocytes.

In order to explain immune-related myocarditis, several hypotheses have been proposed. The theory of the "shared antigen" remains the most plausible mechanism. ${ }^{16}$ In this theory, $\mathrm{T}$ cells might target an antigen shared between the tumor and myocardium. Also, this antigen could be different but homologous. The presence of high titles of musclespecific antigens (desmin and troponin) found in some tumors supports this theory. However, our case lacks important lymphocyte infiltration around tumor and other tissues and this fact does not favor this mechanism. Some previous cases of nivolumab induced myocarditis showed a correlation between tumor response and myocardial damage. Again, the present case is different, because necropsy demonstrated tumoral progression.

The role of $\mathrm{PD} 1$ receptor in the pathogenesis is another point of interest. PD-1 and PD-L1 are expressed in human and murine heart. Also, PD-1 deficiency in mice models genetically predisposed to autoimmunity causes a fatal myocarditis. In several models of $\mathrm{T}$ cell-dependent myocarditis, PD-1 protects against inflammation and myocyte 
damage. ${ }^{31}$ Furthermore, ischaemic-reperfusion rat models showed increased expression of PD-1 and PD-L1 in cardiac myocytes. $^{32}$

PD-1 negative T lymphocytes present greater cytotoxic activity against myocardial cells than PD-1 positive ones. Besides, PD-1 deficiency increases inflammation and cardiac damage mediated by $\mathrm{CD} 8+\mathrm{T}$ cells. ${ }^{2}$ In our patient, $50-60 \%$ of $\mathrm{T}$ lymphocytes were negative for PD-1, and this finding would support a more aggressive course.

To this day, we do not have a record of this $\mathrm{AE}$ and the information is given by reports coming from different hospital experiences. We think it would be interesting to promote a global initiative to join all existing cases and try to obtain a valid conclusions and be able to elaborate accurate guidelines for the standardization of prevention, monitoring, diagnosis and therapeutic management of immune-mediated acute and chronic cardiac toxicity, particularly myocarditis.

We believe that one of the most important challenges in the future is the improvement of preventive measures and early detection of cardiac toxicity by monitoring cardiac damage, as myocarditis mortality is nearly $50 \%$ when it has been established. Since myocarditis usually appears in early period, we believe it would be highly recommended the monitoring of the potential markers from the first cycles. However, although we have suggested some potential markers (TnI, BNP), ECG or TTE, we still do not have any accurate biomarkers available.

Moreover, the development of new immunotherapeutic drugs, such as anti-lymphocyte-activated gene-3 (LAG-3) or anti-T cell Ig and mucin-containing protein 3 (TIM-3), as well as B and T lymphocyte attenuator (BTLA) or $\mathrm{V}$-domain Ig suppressor of $\mathrm{T}$ cell activation (VISTA) blockade, with unknown cardiotoxicity incidence, would be an upcoming challenge for practising physicians.

Throughout this research, a multidisciplinary work lead by oncologists, cardiologists, pathologist and radiologists should be performed to achieve an optimal management of immune-related cardiac AE and reduce its deadly capacity.

\section{Conclusions}

Myocarditis is a rare immune-related $\mathrm{AE}$, which is more frequent in patients who receive ICI combination and without CRF, that usually occurs in the first treatment cycles and causes death in about $50 \%$ of cases, hence the importance of establishing a prevention and monitoring program as well as knowing its underlying molecular mechanisms.

\section{Informed consent and ethical statements}

Written informed consent has been provided by patient's wife for the necropsy and to have the case details published. Institutional approval was not required to publish the case details.

\section{Disclosure}

The authors report no conflicts of interest in this work.

\section{References}

1. Motzer RJ, Escudier B, McDermott D, et al. Nivolumab versus everolimus in advanced renal-cell carcinoma. $N$ Engl J Med. 2015;373(19):1803-1813. doi:10.1056/NEJMoa1510665

2. Varricchi G, Galdiero MR, Marone G, et al. Cardiotoxicity of immune checkpoint inhibitors. ESMO Open. 2017;2:4. doi:10.1136/ esmoopen-2017-000247

3. Nishimura H, Okazaki T, Tanaka Y, et al. Autoimmune dilated cardiomyopathy in PD-1 receptor-deficient mice. Science. 2001;291:319-322. doi:10.1126/science.291.5502.319

4. Okazaki T, Tanaka Y, Nishio R, et al. Autoantibodies against cardiac troponin I are responsible for dilated cardiomyopathy in PD-1-deficient mice. Nat Med. 2003;9:1477-1483. doi:10.1038/nm955

5. Lucas JA, Menke J, Rabacal WA, Schoen FJ, Sharpe AH, Kelley VR. Programmed death ligand 1 regulates a critical checkpoint for autoimmune myocarditis and pneumonitis in MRL mice. $J$ Immunol. 2008;181:2513-2521.

6. Wang J, Okazaki IM, Yoshida T, et al. PD-1 deficiency results in the development of fatal myocarditis in MRL mice. Int Immunol. 2010;22:443-452. doi:10.1093/intimm/dxq026

7. Frigeri M, Meyer P, Banfi $C$, et al. Immune checkpoint inhibitor-associated myocarditis: a new challenge for cardiologists. Can J Cardiol. 2018;34(1):92.e1-92.e3. doi:10.1016/j.cjca.2017.12.015

8. Tajmir-Riahi A, Bergmann T, Schmid M, Agaimy A, Schuler G, Heinzerling L. Life-threatening autoimmune cardiomyopathy reproducibly induced in a patient by checkpoint inhibitor therapy. J Immunother. 2018;41(1):35-38. doi:10.1097/CJI.0000000000000190

9. Norwood TG, Westbrook BC, Johnson DB, et al. Smoldering myocarditis following immune checkpoint blockade. J Immunother Cancer. 2017;5(1):91. doi:10.1186/s40425-017-0296-4

10. Chen Q, Huang DS, Zhang LW, Li YQ, Wang HW, Liu HB. Fatal myocarditis and rhabdomyolysis induced by nivolumab during the treatment of type B3 thymoma. Clin Toxicol (Phila). 2018;56 (7):667-671. doi:10.1080/15563650.2017.1401079

11. Fukusawa Y, Sasaki K, Natsume M, et al. Nivolumab-induced myocarditis concomitant with myasthenia gravis. Case Rep Oncol. 2017;10(3):809-812. doi:10.1159/000479958

12. Matson DR, Accola MA, Rehrauer WM, Corliss RF. Fatal myocarditis following treatment with the PD-1 inhibitor nivolumab. $J$ Forensic Sci. 2018;63(3):954-957. doi:10.1111/15564029.13633

13. Sauer R, Kiewe P, Desole M, et al. Lymphocytic myocarditis in a patient with metastatic clear cell renal cell carcinoma treated with nivolumab. Pathologe. 2017;38(6):535-539. doi:10.1007/ s00292-017-0349-y

14. Tay RY, Blackley E, Mc Lean C, et al. Successful use of equine anti-thymocyte globulin (ATGAM) for fulminant myocarditis secondary to nivolumab therapy. Br J Cancer. 2017;117(7):921-924. doi:10.1038/bjc. 2017.253 
15. Behling J, Kaes J, Münzel T, Grabbe S, Loquai C. New-onset third-degree atrioventricular block because of autoimmune-induced myositis under treatment with anti-programmed cell death-1 (nivolumab) for metastatic melanoma. Melanoma Res. 2017;27(2):155-158. doi:10.1097/CMR.0000000000000314

16. Johnson DB, Balko JM, Compton ML, et al. Fulminant myocarditis with combination immune checkpoint blockade. $N$ Engl J Med. 2016;375(18):1749-1755. doi:10.1056/NEJMoa1609214

17. Tadokoro T, Keshino E, Makiyama A, et al. Acute lymphocytic myocarditis with anti-PD-1 antibody nivolumab. Circ Heart Fail. 2016;9(10). doi:10.1161/CIRCHEARTFAILURE.116.003514

18. Semper H, Muehlberg F, Schulz-Menger J, Allewelt M, Grohé C. Drug-induced myocarditis after nivolumab treatment in a patient with PDL1-negative squamous cell carcinoma of the lung. Lung Cancer. 2016;99:117-119. doi:10.1016/j.lungcan.2016.06.025

19. Gibson R, Delaune J, Szady A, et al. Suspected autoimmune myocarditis and cardiac conduction abnormalities with nivolumab therapy for non-small cell lung cancer. BMJ Case Rep. 2016;2016. doi:10.1136/bcr-2015-212769

20. Kimura T, Fukushima S, Miyashita A, et al. Myasthenic crisis and polymyositis induced by one dose of nivolumab. Cancer Sci. 2016;107(7):1055-1058. doi:10.1111/cas.12961

21. Mehta A, Gupta A, Hannallah F, Koshy T, Reimold S. Myocarditis as an immune-related adverse event with ipilimumab/nivolumab combination therapy for metastatic melanoma. Melanoma Res. 2016;26 (3):319-320. doi:10.1097/CMR.0000000000000251

22. Gill DM, Hahn AW, Hale P, Maughan BL. Overview of current and future first-line systemic therapy for metastatic clear cell renal cell carcinoma. Curr Treat Options Oncol. 2018;19(1):6. doi:10.1007/s11864-018-0517-1

23. Moslehi JJ, Salem JE, Sosman JA, et al. Increased reporting of fatal immune checkpointinhibitor-associated myocarditis. Lancet. 2018;391 (10124):933. doi:10.1016/S0140-6736(18)30302-7

24. Sarocchi M, Grossi F, Arboscello E, et al. Serial troponin for early detection of nivolumab cardiotoxicity in advanced non-small cell lung cancer patients. Oncologist. 2018;Pii:2017-2452.
25. Suzuki S, Ishikawa N, Konoeda F, et al. Nivolumab-related myasthenia gravis with myositis and myocarditis in Japan. Neurology. 2017;89(11):1127-1134. doi:10.1212/WNL.000000000 0004359

26. Suarez-Barrientos A, Wong J, Bell A, Lyster H, Karagiannis G, Banner NR. Usefulness of rabbit anti-thymocyte globulin in patients with giant cell myocarditis. Am J Cardiol. 2015;116(3):447-451. doi:10.1016/j.amjcard.2015.04.040

27. Ammirati E, Oliva F, Belli O, et al. Giant cell myocarditis successfully treated with antithymocyte globuline and extracorporeal membrane oxygenation for 21 days. J Cardiovasc Med (Hagerstown). 2016;17(Suppl 2):e151-e153. doi:10.2459/JCM.00000000000 00250

28. Jain V, Mohebtash M, Rodrigo ME, Ruiz G, Atkins MB, Barac A. Autoimmune myocarditis caused by immune checkpoint inhibitors treated with antithymocyte globulin. J Immunother. 2018;41(7):332-335. doi:10.1097/CJI.0000000000000239

29. Kim HK, Kim KI, Jung SW, et al. Successfully treated acute fulminant myocarditis induced by ulcerative colitis with extracorporeal life support and infliximab. $J$ Cardiovasc Ultrasound. 2016;24 (2):163-167. doi:10.4250/jcu.2016.24.2.163

30. Pérez AR, Fontanella GH, Nocito AL, Revelli S, Bottasso OA. Short treatment with the tumour necrosis factor-alpha blocker infliximab diminishes chronic chagasic myocarditis in rats without evidence of Trypanosoma cruzi reactivation. Clin Exp Immunol. 2009;157(2):291-299. doi:10.1111/j.1365-2249.2009. 03946.x

31. Tarrio ML, Grabie N, Bu DX, Sharpe AH, Lichtman AH. PD-1 protects against inflammation and myocyte damage in $\mathrm{T}$ cell-mediated myocarditis. J Immunol. 2012;188:4876-4884. doi:10.4049/jimmunol.1200389

32. Baban B, Liu JY, Qin X. Upregulation of programmed death-1 and its ligand in cardiac injury models: interaction with GADD153. PLoS One. 2015;10:1-17.

\section{Publish your work in this journal}

Cancer Management and Research is an international, peer-reviewed open access journal focusing on cancer research and the optimal use of preventative and integrated treatment interventions to achieve improved outcomes, enhanced survival and quality of life for the cancer patient.
The manuscript management system is completely online and includes a very quick and fair peer-review system, which is all easy to use. Visit http://www.dovepress.com/testimonials.php to read real quotes from published authors. 